Published in final edited form as:

Science. 2010 October 15; 330(6002): 337-341. doi:10.1126/science.1191968.

\title{
Crystal Growth Inhibitors for the Prevention of L-Cystine Kidney Stones through Molecular Design
}

\author{
Jeffrey D. Rimer ${ }^{\# 1, \dagger}$, Zhihua An ${ }^{\# 1}$, Zina Zhu" ${ }^{\# 1}$, Michael H. Lee ${ }^{1}$, David S. Goldfarb², Jeffrey \\ A. Wesson ${ }^{3}$, and Michael D. Ward ${ }^{1, \ddagger}$ \\ ${ }^{1}$ Department of Chemistry and the Molecular Design Institute, New York University, 100 \\ Washington Square East, New York, NY 10003-6688, USA. \\ ${ }^{2}$ Nephrology Section, New York Harbor VAMC, Department of Urology, St. Vincent's Hospital and \\ NYU School of Medicine, New York, NY \\ ${ }^{3}$ Nephrology Division, Department of Veterans Affairs Medical Center and the Medical College of \\ Wisconsin, 5000 West National Avenue, Milwaukee, WI 53295 \\ \# These authors contributed equally to this work.
}

\begin{abstract}
Crystallization of L-cystine is a critical process in the pathogenesis of kidney stone formation in cystinuria, a disorder affecting at least 20,000 U.S. citizens. Current therapeutic treatments for this disease are somewhat effective but often lead to adverse side effects. Real-time in situ atomic force microscopy (AFM) reveals that L-cystine dimethylester (L-CDME) and L-cystine methylester (LCME) - structural mimics of L-cystine - dramatically reduce the growth velocity of the six symmetry-equivalent $\{100\}$ steps, emanating from well-defined hexagonal spiral dislocations, due to specific binding at the crystal surface that frustrates the attachment of L-cystine solute molecules. Surprisingly, L-CDME and L-CME produce L-cystine crystals with different habits that reveal different binding modes of the two inhibitors at the crystal surfaces. The AFM observations at the nanoscale are mirrored by reduced crystal yield and crystal size in the presence of L-CDME and L-CME, collectively suggesting a new pathway to the prevention of L-cystine stones through the design of crystal growth inhibitors.
\end{abstract}

\section{Keywords}

kidney stone; cystine; crystal growth; cystinuria; atomic force microscopy

Kidney stones comprising L-cystine account for nearly $2 \%$ of adult stones, affecting at least 20,000 patients in the United States. Although this number is substantially smaller than the number of individuals afflicted by calcium oxalate monohydrate (COM) stones (approximately $10 \%$ of the U.S. population), L-cystine stones are larger, recur more

\footnotetext{
‡To whom correspondence should be addressed. mdw3@nyu.edu.

$\dagger$ Present address: Department of Chemical and Biomolecular Engineering S222 Engineering Building, University of Houston 4800 Calhoun Ave. Houston, TX 77204-4004

Supporting Online Material: Materials and Methods, Fig S1 - Fig S10, Table S1, References, and AFM Videos S1-S3.
} 
frequently, and are more likely to cause chronic kidney disease. ${ }^{1}$ The formation of L-cystine stones is a consequence of excessive levels of L-cystine in the urine due to defective reabsorption of filtered cystine, ${ }^{2}$ which in turn is associated with an autosomal recessive disorder caused by mutations in one of the two genes - SLC3A1 on chromosome 2 (type A cystinuria) or SLC7A9 on chromosome 19 (type B cystinuria) - which code for components of the major proximal renal tubule cystine and dibasic amino acid transporter. ${ }^{3}$ This condition is exacerbated by the low solubility of L-cystine, ${ }^{4}$ which favors facile formation of crystals that aggregate into stones, often with centimeter dimensions (Fig 1A).

Current treatments for L-cystine stone prevention include dilution through high fluid intake, ${ }^{5}$ increasing urine $\mathrm{pH}$ through ingestion of alkalinizing potassium or sodium salts, 5,6 or the administration of L-cystine binding thiol drugs (CBTDs) such as D-penicillamine ( $\left.\mathrm{HSC}\left(\mathrm{CH}_{3}\right)_{2} \mathrm{CH}\left(\mathrm{NH}_{2}\right) \mathrm{COOH}\right)$ and a-mercaptopropionylglycine ( $\left.\mathrm{HSCH}\left(\mathrm{CH}_{3}\right) \mathrm{C}(\mathrm{O}) \mathrm{NHCH}_{2} \mathrm{COOH}\right)$, which react with L-cystine to generate more soluble asymmetric disulfides. ${ }^{2}$ These treatments suppress, but typically do not completely prevent, stone formation. CBTDs have an unpleasant odor, can cause adverse side effects such as nausea, fever, fatigue, and skin allergies, ${ }^{5}$ and must be accompanied by high fluid intake to achieve an acceptable cystine excretion rate. ${ }^{5}$ Moreover, these drugs do not reduce L-cystine concentrations sufficiently at dosages (up to $2000 \mathrm{mg}$ /day) regarded as below the threshold for hypersensitivity and toxicity. Herein we report an alternative approach to the prevention of L-cystine kidney stones based on crystal growth inhibition achieved through the binding of tailored growth inhibitors - L-CDME and L-CME - to specific crystal surfaces through molecular recognition as revealed by in situ real-time atomic force microscopy (AFM) $)^{7,8}$ and by parallel bulk crystallization studies.

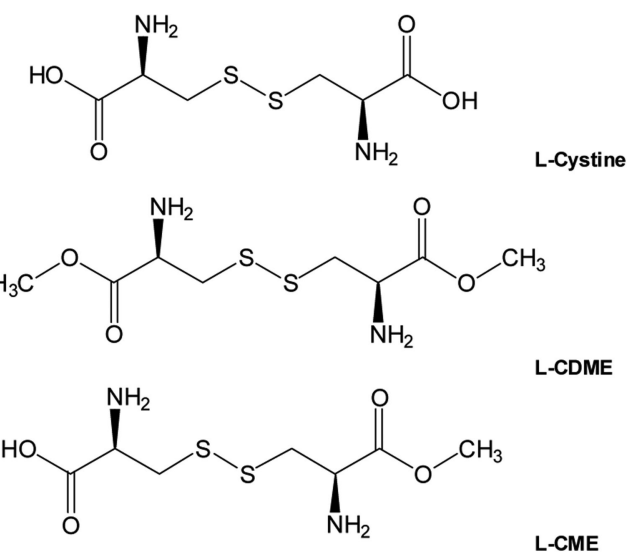

L-cystine stones are aggregates of individual crystals with hexagonal habits (Fig. 1). Lcystine can be crystallized in vitro at physiological $\mathrm{pH}(6 \leq \mathrm{pH} \leq 8)$ by slow evaporation, ${ }^{9}$ acidification of basic L-cystine solutions to neutral $\mathrm{pH},{ }^{10}$ or gradual cooling of solutions supersaturated with L-cystine. ${ }^{11}$ Under these conditions, L-cystine crystallizes as hexagonal plates (Fig. 1B) with large (001) basal surfaces that can achieve widths of $400 \mu \mathrm{m}$ wide and are bounded by six equivalent $\{100\}$ faces. The typical thickness of these crystals ranges from $10-30 \mu \mathrm{m}$. The crystal structure (hexagonal $P 6_{1} 22$ space group, $a=b=0.5422 \mathrm{~nm}, c$ $=5.6275 \mathrm{~nm}$ ) reveals L-cystine molecules organized as a helix about the $6_{1}$ screw axis such 
that six cystine molecules span the $\sim 5.6 \mathrm{~nm}$ unit cell length of the $c$-axis. ${ }^{12}$ The L-cystine molecules exhibit intermolecular $\mathrm{NH}_{3}{ }^{+} \ldots{ }^{-} \mathrm{O}(\mathrm{C}=\mathrm{O})$ hydrogen bonding along the $6_{1}$ screw axis (Fig. 1C, I), intermolecular S...S interactions between the helices at intervals of $c / 2$ along each of the six equivalent $\{100\}$ directions (Fig. 1C, II), and $\mathrm{NH}_{3}{ }^{+} \ldots{ }^{-} \mathrm{O}(\mathrm{C}=\mathrm{O}$ ) hydrogen bonding (Fig. 1D, III, IV) between adjacent helices in the (001) plane. The hexagonal plate habit reflects the multiple strong intermolecular interactions in the (001) plane. The basal surfaces of L-cystine grown at neutral $\mathrm{pH}$ are decorated with $\{100\}$ steps that are observable by either optical or scanning electron microscopy (SEM).

Crystal growth near equilibrium is commonly described by the terrace-ledge-kink model, ${ }^{13}$ wherein steps created by dislocations advance across crystal terraces by the addition of solute molecules to kink sites along the ledge (a ledge is the intersection of a step and terrace). Steps originating from screw dislocations typically exhibit a spiral growth pattern with the first turn occurring once the step reaches a critical length. ${ }^{14}$ Real-time in situ AFM of the L-cystine (001) face during growth in aqueous solutions containing L-cystine revealed steps emanating from screw dislocations, generating hexagonal hillocks in a spiral growth pattern. Occasionally, multiple dislocations were observed (Fig. 2A,B), merging to generate a range of step heights from $1 \mathrm{~nm}$ to $60 \mathrm{~nm}$, with the larger steps observed distant from the dislocation cores where step bunching would be expected. In contrast, hillocks generated by single isolated dislocations were bounded by six well-defined major $\{100\}$ steps, each with a $\sim 6 \mathrm{~nm}$ height corresponding to the unit cell length along $c$, separating (001) terraces. Each hillock terrace was decorated with six minor $\{100\}$ steps at sixty degree intervals, each with a $\sim 1 \mathrm{~nm}$ height corresponding to a single L-cystine molecule, creating the appearance of a pinwheel. These minor steps most likely reflect a splitting of the dislocation into six equivalent dislocations described by a Burgers vector having a magnitude of $c / 6$. Consecutive images during crystal growth revealed a clockwise rotation of the pinwheel at the dislocation core (i.e. a left-handed screw) accompanied by continuous generation of new hillocks. Attachment of L-cystine molecules to both the minor and major steps on the surrounding terraces results in outward advancement of the steps with respect to the dislocation core (see videos, supporting online material). Notably, the spiral growth pattern also is observed for D-cystine, the unnatural enantiomer, but with counterclockwise (i.e. a right-handed screw) rotation of the pinwheel (Fig, 2C,D). To our knowledge, the observation of screw dislocations of opposite handedness for enantiomeric crystals has not been observed previously, although it would be expected and has been predicted. ${ }^{15}$

The spiral hillocks provide an ideal interface for quantitative determination of crystal growth rates at the near-molecular level with AFM through direct measurement of the $\{100\}$ step velocity (Fig. 3A,B). In order to achieve growth rates within a reasonable measurement timeframe the L-cystine concentration was adjusted to $480 \mathrm{mg} / \mathrm{L}$ ( $2 \mathrm{mM}$ ), five times larger than L-cystine solubility at room temperature. ${ }^{16}$ The $\{100\}$ step velocity $\left(\mathrm{V}_{\mathrm{o}}\right)$, determined by measurement of the step advance in successive images acquired at known time intervals, was $11 \mathrm{~nm} / \mathrm{sec}$, equivalent to 50 molecules $/ \mathrm{nm}^{2}$-sec. The step velocity was equivalent along all six directions, as expected for the hexagonal symmetry.

Crystallization outcomes such as habit, chirality, and polymorphism can be influenced by tailored growth inhibitors that reduce crystallization rates through binding at specific step 
sites. ${ }^{17,18}$ These inhibitors, which have been described as "imposters," 14 consist of a binder moiety that emulates a critical structural element of the solute that attaches to a specific crystal site and a perturber moiety that obstructs the approach of additional solute molecules to neighboring sites, thereby pining step motion. Growth inhibitors may be monomers that closely resemble the solute, which has been demonstrated for amino acid crystals. ${ }^{19}$ Polyvalent macromolecules capable of binding to multiple crystal sites, such as peptides and proteins also can influence ice crystallization ${ }^{20}$ and the formation of biominerals, including calcium carbonate ${ }^{21,22}$ and calcium oxalates. ${ }^{23,24,25}$

AFM revealed that addition of L-CDME, a structural mimic of L-cystine in which the carboxylate groups are replaced by methylester groups, resulted in roughening of the otherwise highly linear $\{100\}$ step edges and rounding of the hillock corners, consistent with step pinning through adsorption of L-CDME at the $\{100\}$ steps (Fig. 2E,F). This effect was reversible, as the steps once again become linear following addition of aqueous solutions containing only L-cystine (Fig. S3). The step velocity decreased monotonically with increasing L-CDME concentration, becoming negligible above $30 \mathrm{mg} / \mathrm{L}$ (Fig. 3A,C). Like the step roughening, the inhibitor effect was reversible, with the rates returning to the original value once the growth medium was replaced with aqueous solutions containing only L-cystine. The reduction of the step velocity was equivalent along all six directions in the (001) plane, as expected for the hexagonal symmetry of the crystal. These observations are consistent with attachment of L-CDME to the $\{100\}$ step planes through a combination of intermolecular (cystine)S...S(L-CDME), (cystine) $\mathrm{C}(=\mathrm{O}) \mathrm{O}^{-} . .{ }^{+} \mathrm{H}_{3} \mathrm{~N}(\mathrm{~L}-\mathrm{CDME})$, and (cystine) $\mathrm{N}-\mathrm{H} . . . \mathrm{O}=\mathrm{C}(\mathrm{L}-\mathrm{CDME})$ interactions in a manner that mimics the attachment of $\mathrm{L}$ cystine solute molecules at the $\{100\}$ steps, with the ester methyl groups of bound L-CDME molecules blocking the attachment of L-cystine solute molecules at neighboring crystal sites. Notably, the steric bulk of the ester methyl group is not sufficient to prevent binding to the $\{100\}$ steps.

The AFM measurements demonstrate that L-CDME slows growth along the naturally fast growth directions within the (001) plane. This microscopic behavior was mirrored in bulk crystallization by a gradual change in the crystal habit from (001) plates to small hexagonal needles oriented along the [001] direction as the L-CDME concentration was increased (Fig. $4 \mathrm{~A})$. At L-CDME concentrations as low as $5 \mathrm{mg} / \mathrm{L}(0.02 \mathrm{mM}$; equivalent to $1 \%$ of the $\mathrm{L}$ cystine concentration) the area of the (001) face was reduced nearly 1000-fold whereas the length of the needles was comparable to the thickness of the hexagonal plates grown in the absence of L-CDME $(\sim 30 \mu \mathrm{m})$, resulting in a 1000-fold reduction in crystal volume. Moreover, the total crystallization yields decreased with increasing L-CDME concentration, approaching complete inhibition above $2 \mathrm{mg} / \mathrm{L}$ (Fig. 3D), consistent with the AFM observations. L-CDME also promoted the formation of small amounts of minute crystals of tetragonal L-cystine (Fig. 4B). The formation of the tetragonal polymorph, which ordinarily crystallizes only under more basic conditions $(\mathrm{pH}>8),{ }^{26}$ can be attributed to the suppressed growth of the hexagonal form by L-CDME.

The unsymmetrical L-cystine methylester (L-CME), a cystine mimic with only one ester methyl group, also inhibited L-cystine crystallization. AFM measurements revealed that the $\{100\}$ step velocity declined with increasing L-CME concentration, but to a lesser extent 
than L-CDME (Fig. 3B). L-CME also reduced the size of L-cystine crystals substantially, and it reduced the crystal yield, but to a lesser extent than L-CDME (Fig. 3D). Step roughening was observed in the presence of L-CME, but at higher concentrations compared with L-CDME (Fig. S3). These observations are consistent with weaker inhibition of LCME compared with L-CDME. Interestingly, the L-cystine crystals grown in the presence of L-CME exhibited a tapered hexagonal habit, with six faces intersecting the basal (001) plane at an angle of $85^{\circ}$, attributable to the emergence of six equivalent $\{101\}$ surfaces (Fig. 4C,D). The different crystal habits generated by L-CDME and L-CME provide interesting insight into the binding modes of these inhibitors at the L-cystine crystal surface.

The $\mathrm{P}_{1} 22$ space group symmetry generates inequivalent projections of the L-cystine molecules on each flank of a hexagonal hillock. Each projection winds around the hillock by translations of $\pm c / 6$ on adjacent faces. In the $5.6 \mathrm{~nm}$ span of the hillock, diad axes create two unique pairs of symmetry-related projections in which the L-cystine molecules are oriented in opposite directions along $c$, for example, $\mathrm{C} 1 / \mathrm{C} 3$ and $\mathrm{C} 4 / \mathrm{C} 6$ on the (010) face (Fig. 5). The two remaining sites, located on the diad special positions, create two additional unique projections along directions containing the aforementioned intermolecular $\mathrm{S}$...S contacts. Molecular models reveal that binding of L-CDME at $(001) \cap\{100\}$ ledge sites (illustrated in Fig. 5 by the intersection of (001) and (010) planes) is precluded by the steric obstruction introduced by one of the ester methyl groups (Fig. 5). Consequently, attachment of L-CDME must occur at L-cystine sites above the ledge sites. It is reasonable to suggest that L-CDME would bind preferentially at the diad sites because of the additional S...S interactions, for example, the C2 and C5 sites on the (010) face. Binding of L-CDME would prevent steps from advancing further above the inhibitor site, leading to step bunching that would terminate in $\{100\}$ planes and producing hexagonal needles with small cross sections. LCDME binding to other sites cannot be excluded; the projections of L-cystine molecules at each site of a diad-related pair (i.e. blue-blue or green-green on each hillock face in Fig. 5) differ only with respect to their "up" or "down" orientation (denoted by the white arrows). The $\mathrm{C}_{2}$ symmetry of L-CDME would result in equivalent binding to either site of a diad pair.

In contrast, L-CME can bind at $(001) \cap\{100\}$ ledge sites in its "up" orientation because the carboxylic acid terminus is indistinguishable from the ends of L-cystine (the ester methyl group would prevent binding at the ledge in the "down" orientation). The terrace of the ledge site effectively breaks the twofold "up-down" symmetry of the diad-related pairs.

Consequently, each flank of the hexagonal hillock contains six crystallographically, and therefore chemically, inequivalent $(001) \cap\{100\}$ ledge sites along the $5.6 \mathrm{~nm}$ span of the $c$ axis (Fig. S10). The tapered crystals grown in the presence of L-CME can only be explained by highly selective binding of L-CME at one of these unique sites. Crystal step motion would then be pinned at these sites, repeating at an interval of $c$, creating a vicinal face with a tangent plane intersecting the (001) plane at an angle of $85^{\circ}$, identical to the angle of the tapered faces on the macroscopic crystals and assignable to $\{101\}$ planes. The different mode of binding in the presence of L-CDME and L-CME, particularly the discrimination of L-CME for one of the six possible ledge sites, is a remarkable illustration of molecular recognition between tailored inhibitors and crystal surface sites. Moreover, the step velocity measurements, step roughening, crystal habit effects, and crystal yields represent a rare 
example of a correspondence between inhibitor effects at the microscopic level and macroscopic crystallization behavior.

L-CDME is extraordinarily effective compared with inhibitors examined for other kidney stone forming materials. Molecular additives have been found to be substantially less effective as crystal growth inhibitors of COM compared with their polymeric forms; for example, aspartic acid is more than 1000 times less active for COM inhibition than poly(aspartic acid), in which the carboxylate side chains are thought to bind to calcium sites on the crystal surfaces. The effective inhibition observed for polymers can be attributed to the entropic benefit associated with binding of multiple carboxylate groups on a single chain. Surprisingly, the concentrations at which L-CDME becomes effective for L-cystine inhibition $(\sim 2 \mathrm{mg} / \mathrm{L})$ is comparable to those observed for poly(amino acid) inhibitors of COM crystallization. ${ }^{23}$ The importance of molecular recognition between L-CDME and the L-cystine step planes is underscored by our observations that other additives with proximal carboxylate and amine groups, like those in L-cystine, had a negligible influence on Lcystine growth. For example, L-cysteine, the thiol relative of L-cystine, reduced the size of L-cystine crystals somewhat (at $10 \mathrm{mg} / \mathrm{L}$ ) but its effect on crystallization yield was negligible. Notably, urinary proteins such as osteopontin, human serum albumin and TammHorsfall protein, afforded only modest reductions in crystallization yield at concentrations comparable to physiological values $(2 \mathrm{mg} / \mathrm{L})$, suggesting a negligible role for these substances in the regulation of cystine stone formation.

Collectively, the AFM and bulk crystallization behavior for L-cystine suggest that L-CDME is a viable therapeutic agent for the prevention of L-cystine kidney stones. This promises a unique approach to stone prevention using a benign crystal growth inhibitor at low concentrations rather than drugs that rely on a chemical reaction with L-cystine (i.e. CBTDs), increases in urine alkalinity (which are often accompanied by undesirable side effects), or dramatic increases in urine volume (which can be unreliable owing to patient non-adherance). The reduction in mass yield in the presence of inhibitors is a kinetic effect that maintains a metastable supersaturated L-cystine concentration, but from a pathological perspective this is a sufficient condition for preventing stone formation. L-cystine stone formers typically have urinary L-cystine concentrations ranging from 250 to $1,000 \mathrm{mg} / \mathrm{L}$ (equivalent to 1 - $4 \mathrm{mM}$ ), comparable to the concentrations used for the AFM and bulk crystallization studies described above. Therefore, L-CDME concentrations near $2 \mathrm{mg} / \mathrm{L}$ (< $0.01 \mathrm{mM}$ ), at which inhibition of L-cystine growth was highly effective, will likely be adequate for therapeutic effect. Cell culture data, acquired for the purpose of evaluating cystine exodus from lysosomes, show loss of cell viability at approximately $1 \mathrm{mM} \mathrm{L}$ CDME, and studies in rats, performed to measure oxidative stress in the brain cortex, demonstrated adverse effects at dosages of approximately $500 \mathrm{mg} / \mathrm{kg}$ (rat) per day. ${ }^{27,28,29}$ Although the pharmacokinetics of L-CDME are not well known, a L-CDME dose of 10 - 50 mg per day, far below toxic levels (based on typical daily urine volumes) but greater than the amount needed for crystal growth inhibition in vitro, may prove sufficient to achieve adequate L-CDME concentrations in urine for crystal growth inhibition in vivo. Furthermore, the reduction of L-cystine crystal size in the presence of L-CDME and L-CME reduces the overall surface area available for aggregation and attachment to renal epithelial cells, and the change in crystal habit alters the relative areas of the exposed crystal faces, 
which can have different adhesive properties ${ }^{30}$ SEM cross-sectional images of L-cystine stones reveal stacking of (001) faces of single crystals (Fig S5), and the observed reduction of the area of these faces by L-CDME and L-CME is expected to mitigate crystal aggregation, a critical step in stone formation.

\section{Supplementary Material}

Refer to Web version on PubMed Central for supplementary material.

\section{Acknowledgments}

This work was supported by the National Institutes of Health and the Office of Rare Disease Research (NIDDK R01-DK068551 and 1U54DK083908-01) and the NYU Molecular Design Institute. Use of the Advanced Photon Source was supported by NSF/DOE (CHE-0535644). The authors thank Dr. Chunhua Hu, Dr. Minfeng Li, Dr. YuSheng Chen, and Prof. Glen Kowach for technical assistance, and Prof. Bart Kahr for helpful discussions.

\section{References}

1. Ahmed K, Dasgupta P, Khan MS. Postgrad. Med. J. 2006; 82:799. [PubMed: 17148700]

2. Dolin DJ, et al. J. Endourol. 2005; 19:429. [PubMed: 15865542]

3. Mattoo A, Goldfarb DS. Semin. Nephrol. 2008; 28:181. [PubMed: 18359399]

4. Moe OW. Lancet. 2006; 367:333. [PubMed: 16443041]

5. Becker G. Nephrology. 2007; 12:S4. [PubMed: 17316277]

6. Nakagawa Y, et al. J. Urology. 2000; 164:1481.

7. Hillier AC, Ward MD. Science. 1994; 263:1261. [PubMed: 17817430]

8. Ward MD. Chem. Rev. 2001; 101:1697. [PubMed: 11709996]

9. Dahaoui S, et al. J. Phys. Chem. A. 1999; 103:6240.

10. Girija EK, Kalkura SN, Ramasamy P. J. Mater. Sci. 1995; 6:617.

11. Fujiki Y, Tokunaga N, Shinkai S, Sada K. Angew. Chem. Int. Ed. 2006; 45:4764.

12. Oughton BM, Harrison PM. Acta Cryst. 1959; 12:396. CCDC REFCODE LCYSTI10.

13. Tiller, WA. The Science of Crystallization: Microscopic Phenomena and Defect Generation.

Cambridge University Press; Ithaca, NY: 1991.

14. Sizemore JP, Doherty MF. Cryst. Growth Des. 2009; 9:2637.

15. Levitt LS. Indian J. Phys. 1975; 49:696.

16. Carta R, Tola G. J. Chem. Eng. Data. 1996; 41:414.

17. Weissbuch I, Addadi L, Lahav M, Leiserowitz L. Science. 1991; 253:637. [PubMed: 17772367]

18. Clydesdale G, Hammond RB, Roberts KJ. J. Phys. Chem. B. 2003; 107:4826.

19. Weissbuch I, Lahav M, Leiserowitz L. Cryst. Growth Des. 2003; 3:125.

20. Liou YC, Tocilj A, Davies PL, Jia ZZ. Nature. 2000; 406:322. [PubMed: 10917536]

21. Orme CA, et al. Nature. 2001; 411:775. [PubMed: 11459051]

22. Stephenson AE, et al. Science. 2008; 322:724. [PubMed: 18974348]

23. Guo SW, Ward MD, Wesson JA. Langmuir. 2002; 18:4284.

24. Jung T, et al. Langmuir. 2004; 20:8587. [PubMed: 15379479]

25. Grohe B, et al. J. Am. Chem. Soc. 2007; 129:14946. [PubMed: 17994739]

26. Chaney MO, Steinrauf LK. Acta Cryst. B. 1974; 30:711. CCDC REFCODE LCYSTI11.

27. Kessler A, et al. Neurochem. Res. 2008; 33:737. [PubMed: 17940891]

28. Wilmer MJ, et al. Pediatr. Res. 2007; 62:151. [PubMed: 17597653]

29. Foreman JW, et al. Metabolism. 1987; 36:1185. [PubMed: 3683187]

30. Sheng X, et al. Proc. Natl. Acad. Sci. USA. 2005; 102:267. [PubMed: 15625112] 

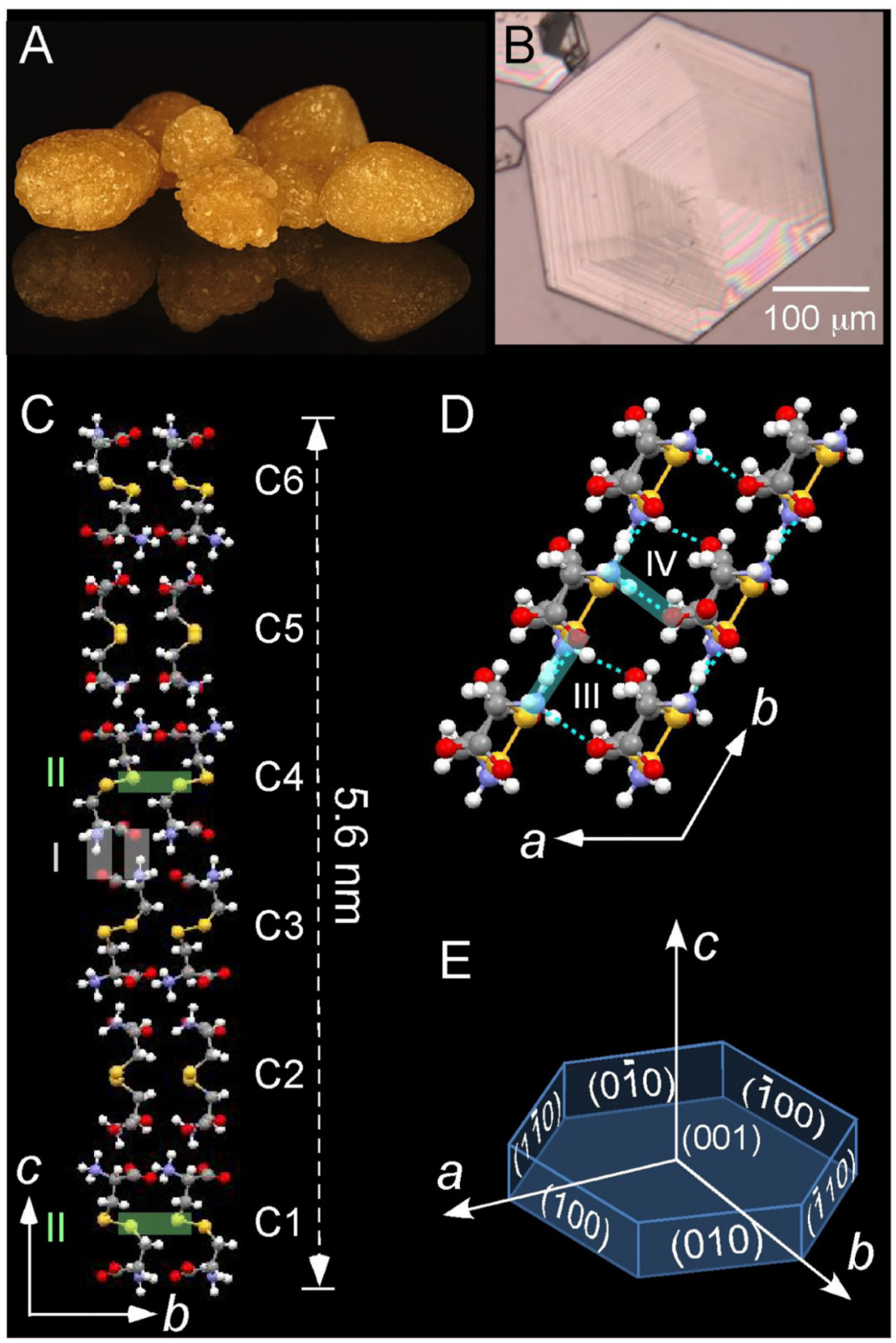

Fig. 1.

(A) Human stones with millimeter-scale dimensions (courtesy of M. Lewis, International Cystinuria Foundation). (B) A hexagonal L-cystine crystal prepared in vitro. (C) Two adjacent helices of L-cystine molecules, viewed on the (100) plane, each winding about a $6_{1}$ screw axis that coincides with the $c$ axis. Six L-cystine molecules, denoted C1 to C6, span the $5.6 \mathrm{~nm}$ c axis. Key intermolecular interactions include amine-carboxylate hydrogen bonds along the helix $\left(\mathrm{I}, d_{\mathrm{N}} \ldots \mathrm{O}=2.87 \AA\right.$ ) and $\mathrm{S}$...S interactions $\left(\mathrm{II}, d_{\mathrm{S} . . . \mathrm{S}}=3.47 \AA\right.$ ) between helices at intervals of $c / 2$, depicted here for $\mathrm{C} 1$ and $\mathrm{C} 4$ along the [010] direction (identical S...S interactions occur at symmetry-related sites along the other five equivalent directions. (D) Intermolecular amine-carboxylate hydrogen bonds in the (001) plane (III, $d_{\mathrm{N}} \ldots \mathrm{O}=2.79$ $\AA$; $\mathrm{IV}, d_{\mathrm{N} . . . \mathrm{O}}=2.81 \AA$ ). Atom color code: carbon (gray), oxygen (red), nitrogen (blue), sulfur 
(yellow), hydrogen (white). (E) Schematic illustration of a hexagonal L-cystine crystal, with Miller indices. The six planes flanking (001) belong to the $\{100\}$ family. 

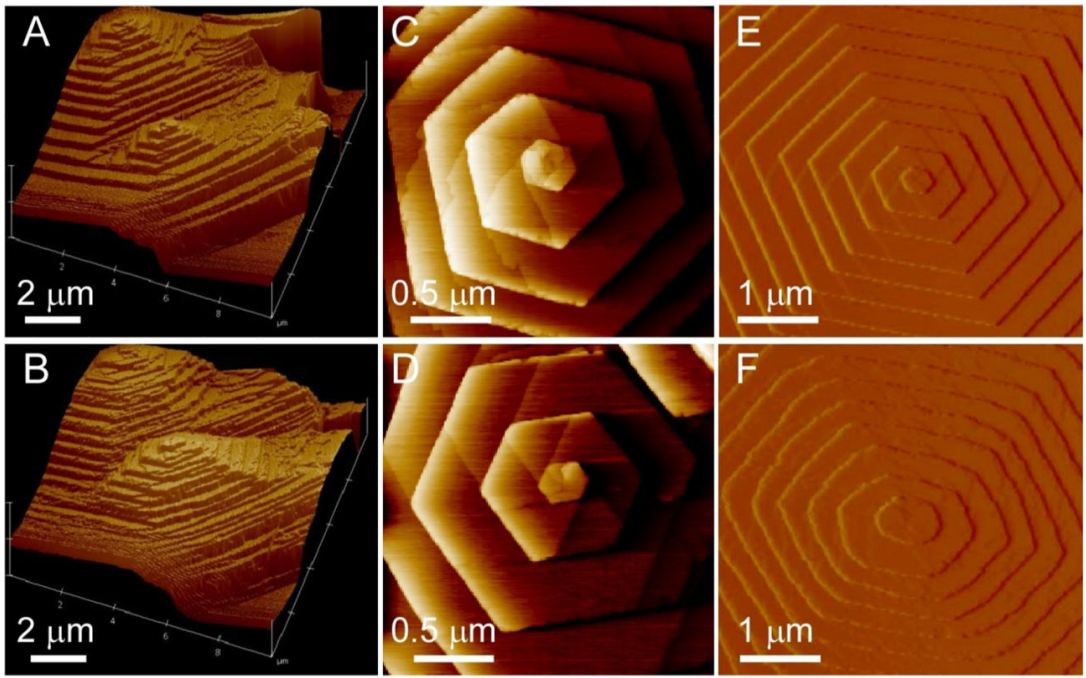

$\mathrm{F}$

Fig. 2.

(A,B) Real-time in situ AFM images of a L-cystine crystal, acquired 12 minutes apart. A pair of hexagonal hillocks generated by two closely spaced dislocations that serve as landmarks to illustrate visualization of hillock growth in still images (see video, supporting online information). (C,D) AFM images of a single dislocation center of (C) L-cystine and (D) D-cystine crystal during growth. (E,F) AFM image of a hexagonal growth hillock on the (001) face of L-cystine before and after addition of L-CDME ( $5 \mathrm{mg} / \mathrm{L} ; 0.02 \mathrm{mM})$, revealing roughening of the $\{100\}$ steps due to step pinning. All images were acquired in aqueous solutions containing $2 \mathrm{mM}$ L-cystine. 

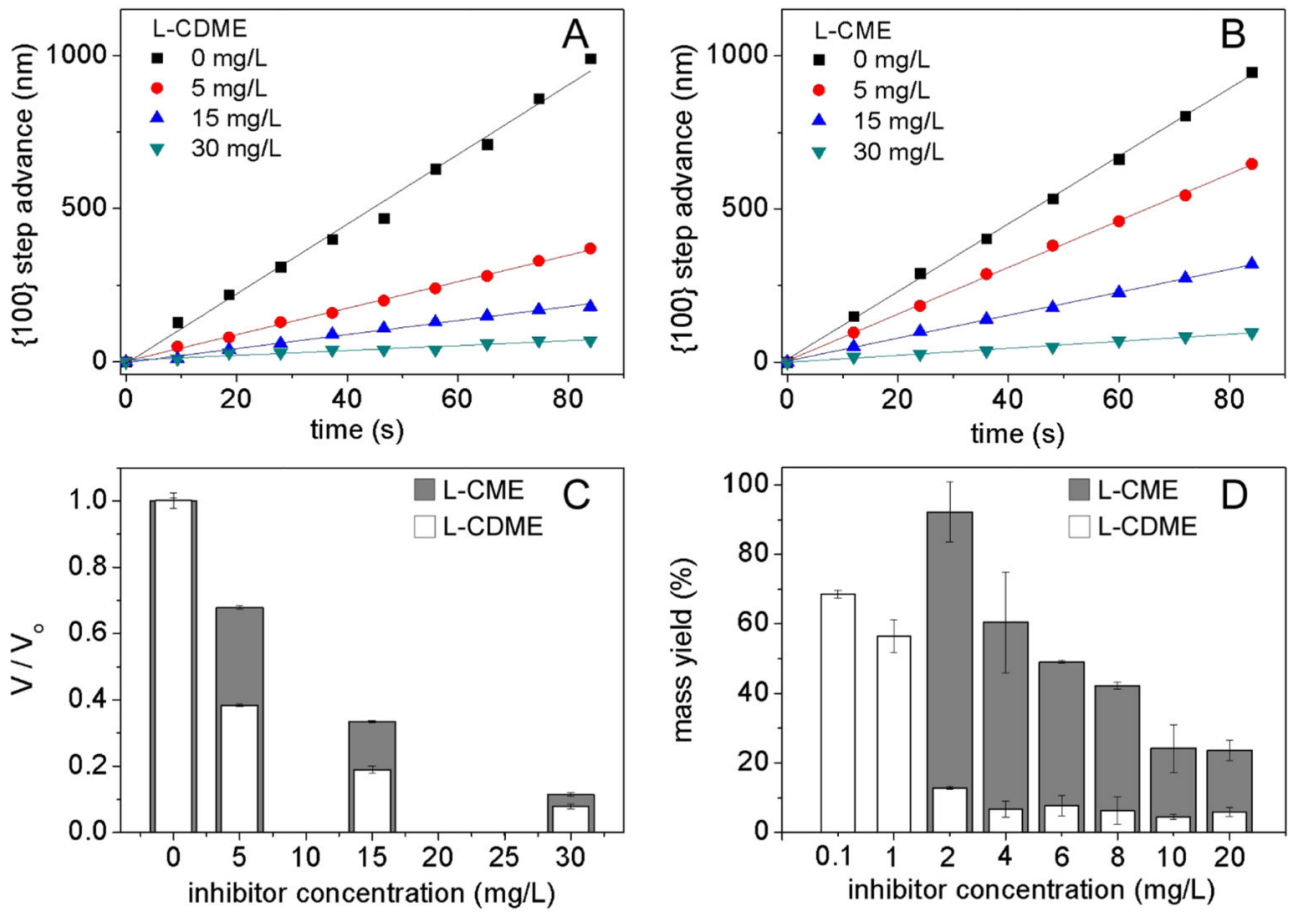

Fig. 3.

(A,B) The position of the $5.6 \mathrm{~nm}$-high $\{100\}$ steps, as measured by real time in situ AFM from the center of the spiral dislocations during growth in aqueous solutions containing 2 $\mathrm{mM}$ L-cystine with various concentrations of L-CDME or L-CME. The step velocities in the absence of inhibitor, determined from the slopes of the lines, are $\mathrm{V}_{\mathrm{o}}=11.3 \pm 0.2$ and 11.4 $\pm 0.1 \mathrm{~nm} / \mathrm{sec}$, respectively. (C) Comparison of the effectiveness of L-CDME and L-CME on the inhibition of the $\{100\}$ step velocity, expressed as $V / V_{0}$ to account for the slight difference in $\mathrm{V}_{\mathrm{o}}$ for different crystals. (D) The total mass of L-cystine crystals obtained after crystallization for 72 hours in the presence of various concentrations of L-CDME or L-

CME. The error bars represent one standard deviation. 

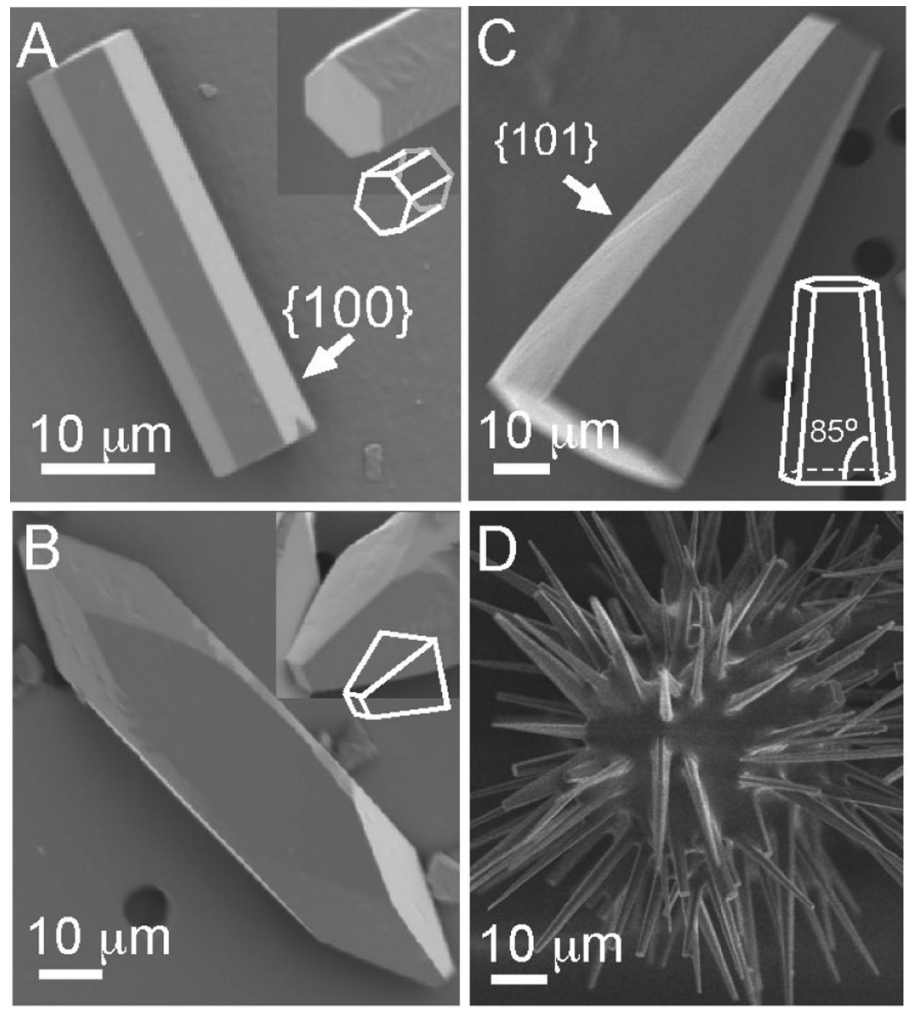

Fig. 4.

(A) Minute L-cystine crystals grown in the presence of L-CDME ( $5 \mathrm{mg} / \mathrm{L} ; 0.02 \mathrm{mM})$ exhibit a hexagonal needle-like habit with prominent $\{100\}$ faces and high $c / a$ aspect ratios, approaching 30 for many crystals. (B) Small quantities of the tetragonal $P 4_{1}$ polymorph are formed in the presence of L-CDME (5 mg/L). (C) L-CME (10 mg/L; $0.04 \mathrm{mM})$ produces tapered hexagonal needles with six $\{101\}$ faces. Some crystals exhibit the tapered habit at both ends of the crystal, as expected for the crystal symmetry (see supporting online material). The observation of only one-half of the tapered crystal suggests growth often begins on a surface. (D) The tapered needles formed in the presence of $10 \mathrm{mg} / \mathrm{L} \mathrm{L}-\mathrm{CME}$ occasionally grow from a nidus that may be an amorphous L-cystine particle or a microscopic unidentified foreign object. 


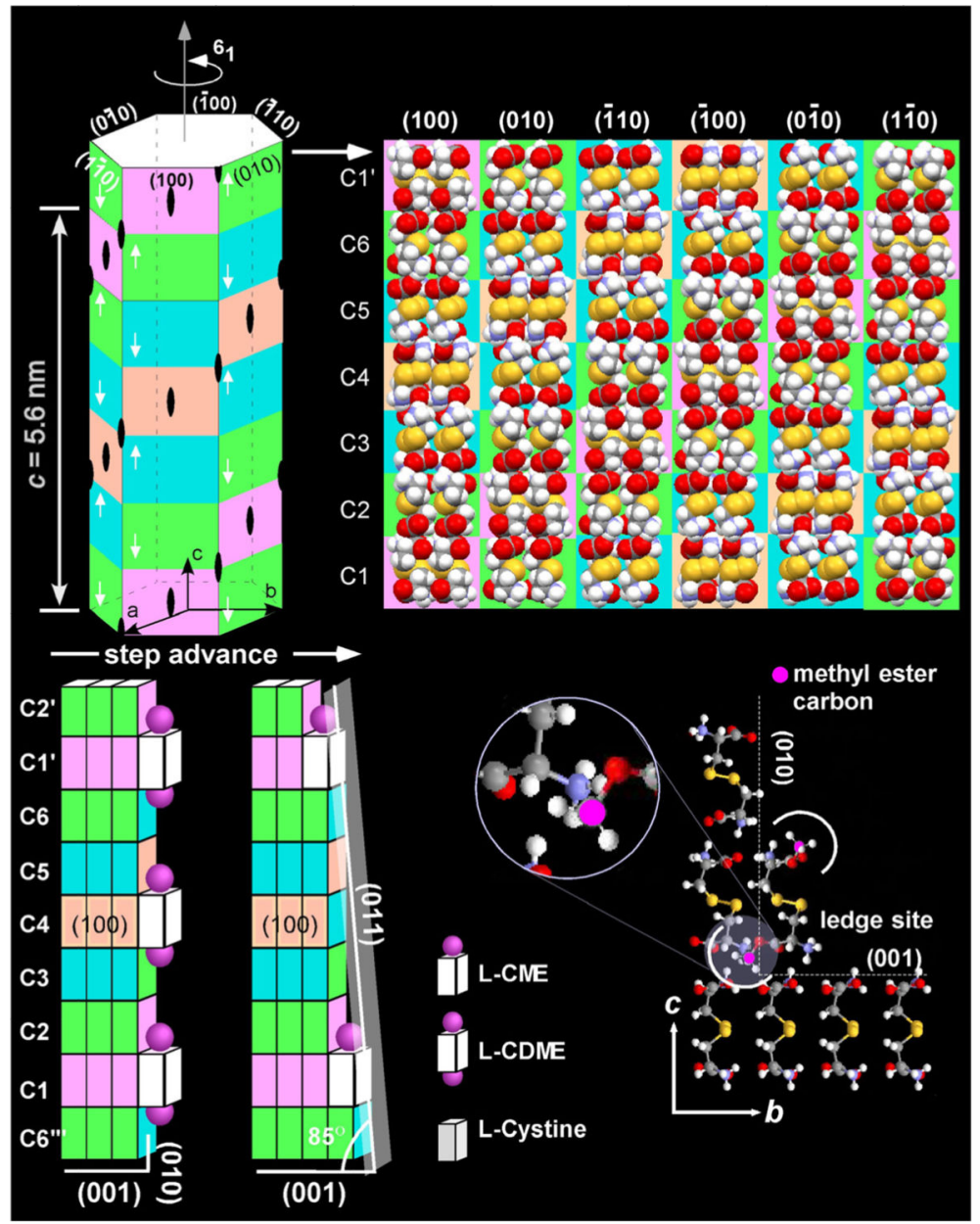

Fig. 5.

(upper left) Schematic representation of an L-cystine hillock, color-coded to denote the distinct projections of L-cystine along each flank of the $5.6 \mathrm{~nm}$-high hillock. The six flanks are depicted in the unraveled version of the hillock on the right. Equivalent projections on adjoining flanks are related by the $6_{1}$ screw axis. Diad axes, denoted by black ovals, generate two identical projections that differ only with respect to their "up" or "down" orientation (denoted by white arrows). (lower right) L-CDME binding at a (001)ก\{010\} ledge site is frustrated by one of the ester methyl group, emphasized here by the white arc. (lower left) LCDME binding to the (010) step: The ester methyl groups of L-CDME, depicted by purple spheres, block attachment of L-cystine to adjacent sites. Binding to the $\mathrm{C} 1$ and $\mathrm{C} 4$ sites on the $\{010\}$ step allows S...S and hydrogen bonding interactions between L-cystine molecules projecting from the step surface and L-CDME. In contrast, in the "up" orientation the carboxylic acid terminus of L-CME can bind to the ledge sites in a manner like L-cystine, through hydrogen bonding interaction I, at the (001) terrace, as well as S...S interactions and hydrogen bonding at the (010) step. Crystallographically equivalent $(001) \cap(010)$ ledge sites, depicted here for the C6"-C1 and C6-C1' combinations, are spaced at intervals of $c$, generating a vicinal surface equivalent to a (011) plane. This plane intersects the (001) plane at an angle of $85^{\circ}$, consistent with the tapered faces observed in the presence of L-CME. 